\title{
Caracterização de cultivares de amendoim quanto ao dano de Stegasta bosquella (Chambers, 1875) (Lepidoptera: Gelechiidae)
}

\author{
Characterization of peanut cultivars to damage Stegasta bosquella \\ (Chambers, 1875) (Lepidoptera: Gelechiidae)
}

\author{
Luciano Nogueira', Flavio Gonçalves de Jesus², André Cirilo de Sousa Almeida ${ }^{2 *}$, Arlindo Leal Bioça Junior ${ }^{1}$, \\ Ignácio José de Godoy ${ }^{3}$, Fernanda Corrêa²
}

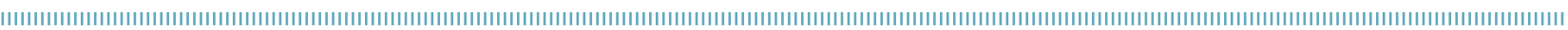

RESUMO: Avaliou-se a resistência de quatro cultivares de amendoim de hábito de crescimento rasteiro e cinco cultivares de hábito de crescimento ereto ao dano de Stegasta bosquella (Chambers, 1875) (Lepidoptera: Gelechiidae) em condiçôes de campo na safra das águas, nos anos 2009/2010 e 2010/2011. Os cultivares utilizados nos experimentos foram: IAC 147, IAC 213, IAC Caiapó e IAC Runner 886 (porte rasteiro) e IAC Tatu ST, IAC 5, IAC 8112, IAC 22 e IAC 88-1 (porte ereto). O delineamento adotado foi em blocos ao acaso, com parcelas subdivididas ao longo do tempo, e quatro repetiçóes. Para a infestação de $S$. bosquella, foram realizadas avaliaçóes semanais dos 18 aos 67 dias após a emergência (DAE), com contagem de lagartas e avaliaçôes de danos. Para a contagem do número de brotos por planta, coletaram-se 5 plantas por parcela a partir dos 30, 60 e 90 DAE. Independentemente do cultivar, as maiores infestaçôes de S. bosquella ocorrem dos 53 aos 67 DAE. O cultivar IAC Runner 886 apresenta a maior emissão de brotos apicais, uma característica desejável na defesa da planta em relação à $S$. bosquella.

PALAVRAS-CHAVE: Arachis hypogaea; lagarta-do-pescoço-vermelho; resistência de plantas.
ABSTRACT: We evaluated the strength of four growth habit of peanut cultivars creeping and five cultivars of erect growth habit to damage Stegasta bosquella (Chambers, 1875) (Lepidoptera: Gelechiidae) under field conditions during the rainy season, in the years 2009/2010 and 2010/2011. The cultivars used in the experiments were: IAC 147, IAC 213, IAC Caiapó and IAC Runner 886 (runner) and IAC Tatu ST, IAC 5, IAC 8112, IAC 22 e IAC 88-1 (erect). The design adopted was randomized blocks with split plot over time, and four replications. For infestation of $S$. bosquella, weekly evaluations were done 18 to 67 days after emergence (DAE), with caterpillars count and damage assessments. For counting the number of shoots per plant, they collected up five plants per plot from the 30, 60 and 90 DAE. Regardless of cultivating major infestations of $S$. bosquella occur from 53 to 67 DAE. The IAC Runner 886 cultivar has the highest emission apical shoots a desirable feature in plant defense in relation to $S$. bosquella.

KEYWORDS: Arachis hypogaea; red-necked peanutworm; host plant resistance. 


\section{INTRODUÇÃO}

$\mathrm{O}$ amendoinzeiro Arachis hypogaea é infestado por numerosas pragas que causam danos desde a alimentação acidental até a total destruição da planta e perdas na produção (GABRIEL et al., 1996). Atualmente, a lagarta do pescoço vermelho - Stegasta bosquella (Chambers, 1875) (Lepidoptera: Gelechiidae) —é considerada a mais importante lepidóptera praga dessa cultura, pelos prejuízos causados e pela ocorrência generalizada (GAllo et al., 2002; BoiçA Junior et al., 2011).

Os sintomas provocados pela infestação dessa praga são bastante típicos e têm sido descritos por diversos autores. Os folíolos novos atacados, ao se abrirem, exibem lesôes simétricas bem características. Em folíolos ainda fechados encontram-se lesôes superficiais e furos, geralmente rodeados de detritos escuros produzidos pela própria lagarta (Sichmann, 1963; Boiça Junior et al., 2011). Com esse ataque, a planta atrasa o desenvolvimento e emite novas brotaçôes (Bondar, 1928; BrandÃo FilHo, 1943). Infestaçôes generalizadas provocam perdas de produtividade, e seu ataque pode ocorrer em qualquer período do desenvolvimento da planta, sendo que no início da cultura merece mais atenção (CRUz et al., 1962).

$\mathrm{Na}$ busca pela redução dos riscos e impactos sobre a saúde humana e ao meio ambiente, causados pelo uso de agrotóxicos, métodos alternativos para o controle de insetos nessa cultura vêm sendo estudados (CHAGas FilHo et al., 2008; BOIÇA JUNiOR et al., 2011).

A resistência de plantas é um deles. Essa técnica é considerada um método ideal, uma vez que apresenta vantagens sobre as demais, não requerendo tecnologia sofisticada por parte dos agricultores. Além disso, as populaçóes podem ser reduzidas abaixo do nível de dano econômico, diminuindo, assim, a poluição ambiental provocada pelos agrotóxicos (LARA, 1991; JANini, 2009; BoIÇA JUNIOR et al., 2012).

De modo geral, plantas de amendoim com resistência podem reduzir de 10 a $35 \%$ os danos causados por insetos-pragas em relação a um cultivar suscetível. Uma planta com moderada resistência pode representar de 35 a $65 \%$ de redução de danos e uma planta com alta resistência mostrará reduções superiores a 65\% (CAMPBELL; Wynne, 1980). Di Bello et al. (2015) concluíram que os cultivares de hábito de crescimento rasteiro, IAC 147 e IAC Runner 886, e os cultivares IAC 22 e IAC 8112 , de hábito de crescimento ereto, apresentaram resistência do tipo antibiose à $S$. bosquella. Boiça Júnior et al. (2013) concluíram que o cultivar de amendoim de hábito de crescimento ereto IAC Tatu ST foi menos consumida por lagartas de Spodoptera cosmioides.

Assim, o objetivo deste trabalho foi avaliar o comportamento de quatro cultivares de amendoim de hábito de crescimento rasteiro e cinco cultivares de hábito de crescimento ereto ao dano de $S$. bosquella em condiçóes de campo.

\section{MATERIAL E MÉTODOS}

Os experimentos foram instalados em condiçôes de campo, na safra das águas, nos anos agrícolas de 2009/2010 e 2010/2011 no município de Urutaí (GO) (17 27’ S; 48 12’ W e 800 m altitude), em Latossolo Vermelho Distroférrico (EMBRAPA, 1999). O clima predominante na região é tropical semiúmido, que se caracteriza por temperatura média de $23^{\circ} \mathrm{C}$, com primavera e verão chuvosos e inverno seco, com precipitação pluviométrica anual entre 1.500 e $1.800 \mathrm{~mm}$.

Para cada experimento, adotaram-se 4 cultivares de hábito de crescimento rasteiro (IAC 147, IAC 213, IAC Caiapó e IAC Runner 886) e 5 ereto (IAC Tatu ST, IAC 5, IAC 8112, IAC 22 e IAC 88-1). O espaçamento adotado foi de $0,90 \mathrm{~m}$ entre linhas para os cultivares de hábito de crescimento rasteiro e 0,60 m entre linhas para as de porte ereto, com 15 sementes por $\mathrm{m}$ linear. As sementes foram previamente tratadas com fungicida (Thiram — 144 g.i.a/100 kg de semente). Após 12 dias do plantio, realizou-se o desbaste, deixando-se 11 plantas por $\mathrm{m}$ linear. $\mathrm{Na}$ adubação de plantio, utilizou-se a quantidade de 250 kg.ha- ${ }^{-1}$ da fórmula $05-25-15$. Os demais tratos culturais foram realizados quando necessários e de acordo com as recomendações de Santos (2005).

\section{Avaliação da população de Stegasta bosquella}

Para a avaliação da infestação de $S$. bosquella, foram realizadas coletas semanais dos 18 aos 67 dias após a emergência (DAE) das plantas, coletando 10 brotos apicais por parcela para contagem do número de lagartas. Para atribuiçôes das notas de danos foliares, foram avaliados dez folíolos semiabertos por parcela, do terço médio das plantas, e atribuídas as notas variando de 1 a 5 , em que a nota 1 representa folíolo sem sintoma de ataque, e 5 , folhas totalmente danificadas pela lagarta, de acordo com JANINE (2009).

\section{Contagem do número de brotos apicais}

Aos 30, 60 e 90 DAE, foram selecionadas ao acaso 5 plantas por parcela para a contagem do número de brotos por planta, de acordo com Lourençấo et al. (2007).

O delineamento experimental foi em blocos ao acaso, empregando-se um esquema de parcela subdividida ao longo do tempo (nove cultivares com oito épocas de avaliação, para a infestação de $S$. bosquella, e nove cultivares com três épocas de avaliação, para a quantidade de brotos apicais) em quatro repetiçôes. Cada unidade experimental constituiu-se de cinco linhas de $5 \mathrm{~m}$ de comprimento. As duas linhas laterais e $0,5 \mathrm{~m}$ iniciais e finais de cada linha foram consideradas como bordadura. Dessa forma, as duas linhas centrais foram deixadas para avaliação do experimento, sendo duas delas para as avaliaçóes da infestação das pragas e contagem do número de 
brotos apicais. Os dados coletados foram submetidos à análise de variância pelo teste $\mathrm{F}$ e as médias foram comparadas pelo teste de Tukey $(\mathrm{p}<0,05)$.

\section{RESULTADOS E DISCUSSÃO}

Os números médios da infestaçáo de $S$. bosquella nos diferentes cultivares de amendoim não diferiram estatisticamente entre si, tanto na safra 2009/2010 como 2010/2011 (Tabela 1). Já para as diferentes épocas de avaliaçôes foram observadas, nas duas safras, diferenças entre um intervalo e outro.

De maneira geral, analisando a infestação de $S$. bosquella nas safras 2009/2010 e 2010/2011, o cultivar IAC 147 apresentou as menores infestaçóes da praga em ambas as safras. Já IAC 22 comportou-se de forma contrária, com maiores infestações.

Comparando-se as diferentes épocas de avaliação após o plantio e as notas de danos, nas duas safras, observou-se que as maiores infestaçóes de $S$. bosquella nos cultivares ocorreram dos 53 aos $67 \mathrm{DAE}$, e as menores, dos 18 aos $46 \mathrm{DAE}$, para as safras de 2009/2010 e 2010/2011. Comparando-se os dados da infestação com as notas de danos, observou-se que a predominância de maiores danos é quantificada quando se tem as maiores infestaçóes de $S$. bosquella.

Para a interação cultivares versus época de avaliação na safra 2009/2010 (Tabela 2), foram verificadas diferenças aos 53, 60 e $67 \mathrm{DAE}$ em relação à infestação de S. bosquella. Aos $53 \mathrm{DAE}$, o cultivar IAC Caiapó apresentou o maior valor em relação à infestação da praga, enquanto IAC 213, IAC Runner 886, IAC 147, IAC 22 e IAC 88-1 exibiram os menores. Aos 60 DAE, as maiores médias foram visualizadas em IAC 8112, e os cultivares IAC 147, IAC 213 e IAC 5 destacaram-se dos demais por não apresentarem infestaçâo da praga. Aos $67 \mathrm{DAE}$, os maiores índices foram em IAC 88-1, e os menores, em IAC Caiapó, IAC, IAC Tatu ST e IAC 5, respectivamente, que também não apresentaram a presença da praga nessa avaliação.

JANINI (2009), estudando a resistência de genótipos de amendoim a $S$. bosquella, encontrou dados diferentes a este, em que foram visualizados maiores infestações e danos nos cultivares IAC Caiapó e IAC Runner 886, quando comparados com espécies silvestres do gênero Arachis. Essas divergências quanto à infestaçáo de $S$. bosquella nos cultivares, segundo LARA (1991), devem ser consideradas, pois a resistência da planta em relação ao inseto é sempre relativa, havendo

Tabela 1. Número e dano médio de Stegasta bosquella por folíolo em nove cultivares de amendoim, em oito épocas, durante o ciclo das plantas, sob infestação natural em campo. Urutaí (GO), 2009/2010 e 2010/2011.

\begin{tabular}{|c|c|c|c|c|}
\hline \multirow{2}{*}{ Cultivar (C) } & \multicolumn{2}{|c|}{ Número de lagartas } & \multicolumn{2}{|c|}{ Nota de dano } \\
\hline & $2009 / 2010$ & $2010 / 2011$ & $2009 / 2010$ & $2010 / 2011$ \\
\hline \multicolumn{5}{|l|}{ Porte Rasteiro } \\
\hline IAC 147 & 0,15 & 0,10 & 1,10 & 1,09 \\
\hline IAC 213 & 0,18 & 0,46 & 1,21 & 1,28 \\
\hline IAC Caiapó & 0,31 & 0,34 & 1,31 & 1,12 \\
\hline IAC Runner 886 & 0,25 & 0,40 & 1,12 & 1,09 \\
\hline \multicolumn{5}{|l|}{ Porte Ereto } \\
\hline IAC Tatu ST & 0,34 & 0,65 & 1,15 & 1,21 \\
\hline IAC 5 & 0,34 & 0,56 & 1,18 & 1,15 \\
\hline IAC 8112 & 0,28 & 0,46 & 1,12 & 1,15 \\
\hline IAC 22 & 0,50 & 0,21 & 1,31 & 1,28 \\
\hline IAC 88-1 & 0,62 & 0,25 & 1,09 & 1,12 \\
\hline$F(C)$ & $1,29^{\mathrm{NS}}$ & $1,48^{\mathrm{NS}}$ & $0,7^{\mathrm{NS}}$ & $0,60^{N S}$ \\
\hline \multicolumn{5}{|c|}{ Época de avaliação (D) } \\
\hline $18 \mathrm{DAE}$ & $0,11 b$ & $0,33 a b$ & $1,00 \mathrm{~b}$ & $1,05 b$ \\
\hline 25 DAE & $0,22 a b$ & $0,38 a b$ & $1,05 b$ & $1,13 b$ \\
\hline $32 \mathrm{DAE}$ & $0,22 a b$ & $0,36 a b$ & $1,11 \mathrm{~b}$ & $1,11 \mathrm{~b}$ \\
\hline 39 DAE & $0,13 b$ & $0,16 b$ & $1,08 b$ & $1,13 b$ \\
\hline $46 \mathrm{DAE}$ & $0,11 b$ & $0,33 a b$ & $1,19 b$ & $1,25 a b$ \\
\hline $53 \mathrm{DAE}$ & $0,61 a$ & $0,41 a b$ & $1,25 b$ & $1,22 a b$ \\
\hline $60 \mathrm{DAE}$ & $0,61 \mathrm{a}$ & $0,52 a b$ & $1,30 a b$ & $1,44 a$ \\
\hline $67 \mathrm{DAE}$ & $0,66 a$ & $0,61 a$ & $1,61 \mathrm{a}$ & $1,05 b$ \\
\hline$F(D)$ & $5,32^{* *}$ & $2,21^{*}$ & $7,36^{* *}$ & $3,93^{*}$ \\
\hline$F(C \times D)$ & $2,14^{* *}$ & $0,88^{\text {NS }}$ & $0,9^{\text {NS }}$ & $0,87^{\mathrm{NS}}$ \\
\hline CV (\%) & 35,24 & 40,79 & 14,68 & 13,57 \\
\hline
\end{tabular}

Médias seguidas de mesma letra não diferem pelo teste de Tukey a $5 \%$ de probabilidade (dados foram transformados em $(x+0,5)^{1 / 2}$, para análise); ** significativo a $1 \%$ de probabilidade; *significativo a $5 \%$ de probabilidade; ${ }^{\text {Ns: }}$ não significativo.

DAE: dias após a emergência. 
necessidade de comparaçáo com outros cultivares, e ocorre em determinadas condiçóes, podendo ou não ser mantida em outras regióes. BoiçA Junior et al. (2012) observaram que esse cultivar consegue suportar o dano de Enneothrips flavens, de modo a apresentar menor redução na produtividade, indicando possuir resistência do tipo tolerância, uma característica importante na defesa da planta em relaçáo à infestação de $S$. bosquella.

Analisando o número de brotos nos cultivares nas duas safras (Tabela 3), verificaram-se diferenças tanto para os cultivares como para as diferentes épocas de avaliação.

Na safra 2009/2010, o cultivar IAC Runner 886 apresentou o maior valor para emissão de brotos, porém sem diferir de IAC 147. Já IAC 22 apresentou menor valor para esse parâmetro, sem diferir de IAC 8112 e IAC 5. Na safra 2010/2011, IAC Runner 886 também apresentou maior valor para emissão de brotos apicais, mas não diferiu de IAC 22, IAC 8112, IAC Caiapó e IAC 5. Os menores índices foram observados em IAC 147 e IAC 213, que não diferiam dos demais, exceto IAC Runner 886.

Em relaçáo às épocas de avaliaçáo, tanto na safra 2009/2010 como na 2010/2011, foram verificados os maiores valores para emissão de brotos por planta aos 60 DAE e declínio na época seguinte (Tabela 3).

Os valores da análise de desdobramento do número médio de brotos por planta entre os cultivares versus as épocas de avaliação são apresentados na Tabela 4. Para a safra 2009/2010, verificou-se diferença entre os cultivares nas três épocas de avaliação.

Aos 30 DAE, a maior emissão de brotos apicais foi observada em IAC 147 e IAC Runner 886, que diferiram dos demais, exceto IAC Caiapó. Já os cultivares de porte ereto apresentaram os menores valores e não diferiram entre si. Aos $60 \mathrm{DAE}$, IAC 147 continuou com a maior emissáo de brotos apicais, e
IAC 22, com a menor. Já aos 90 DAE, IAC Runner 886 apresentou maior emissão de brotos, porém com declínio, quando comparado com as avaliaçóes anteriores, e IAC 22, o menor valor para emissáo de brotos apicais.

Na safra 2010/2011, para a mesma condição avaliada, os resultados foram próximos aos da safra anterior (Tabela 5).

Tabela 3. Número médio de brotos por planta em nove cultivares de amendoim, em três épocas durante o ciclo das plantas, sob infestação natural de Stegasta bosquella em campo. Urutaí (GO), 2009/2010 e 2010/2011.

\begin{tabular}{|c|c|c|}
\hline \multirow{2}{*}{ Cultivar (C) } & \multicolumn{2}{|c|}{ Número de brotos/planta } \\
\hline & $2009 / 2010$ & $2010 / 2011$ \\
\hline \multicolumn{3}{|l|}{ Porte Rasteiro } \\
\hline IAC 147 & $2,73 a b$ & $1,23 \mathrm{c}$ \\
\hline IAC 213 & $1,85 \mathrm{~cd}$ & $1,25 c$ \\
\hline IAC Caiapó & $2,14 b c$ & $1,51 a b c$ \\
\hline IAC Runner 886 & $2,45 \mathrm{a}$ & $1,66 a$ \\
\hline \multicolumn{3}{|l|}{ Porte Ereto } \\
\hline IAC Tatu ST & 1,67 de & $1,29 \mathrm{bc}$ \\
\hline IAC 5 & $1,49 \mathrm{efg}$ & $1,61 a b$ \\
\hline IAC 8112 & $1,35 \mathrm{fg}$ & $1,39 a b c$ \\
\hline IAC 22 & $1,25 \mathrm{~g}$ & $1,36 a b c$ \\
\hline IAC 88-1 & 1,55 def & $1,27 b c$ \\
\hline$F(C)$ & $49,72 * *$ & $5,03 * *$ \\
\hline \multicolumn{3}{|c|}{ Épocas de avaliação (D) } \\
\hline $30 \mathrm{DAE}$ & $1,84 b$ & $1,74 b$ \\
\hline $60 \mathrm{DAE}$ & $2,68 a$ & $2,03 a$ \\
\hline $90 \mathrm{DAE}$ & $0,84 \mathrm{c}$ & $0,42 c$ \\
\hline$F(D)$ & $1007,73 * *$ & 830,61 ** \\
\hline$F(C \times D)$ & $28,61^{* *}$ & 3,71 ** \\
\hline C.V (\%) & 12,10 & 17,78 \\
\hline
\end{tabular}

Médias seguidas de mesma letra não diferem pelo teste de Tukey a $5 \%$ de probabilidade; ** significativo a $1 \%$ de probabilidade; ${ }^{\text {Ns: não }}$ significativo.

DAE: dias após a emergência.

Tabela 2. Valores da análise de desdobramento da interação entre cultivares de amendoim versus épocas de avaliação, referentes ao número médio de Stegasta bosquella por folíolo, sob infestação natural em campo. Urutaí (GO), 2009/2010.

\begin{tabular}{|c|c|c|c|c|c|c|c|c|c|}
\hline \multirow{2}{*}{ Cultivar (C) } & \multicolumn{8}{|c|}{ Épocas de avaliação (D) } & \multirow{2}{*}{$F(C)$} \\
\hline & 18 & 25 & 32 & 39 & 46 & 53 & 60 & 67 & \\
\hline \multicolumn{10}{|l|}{ Porte Rasteiro } \\
\hline IAC 147 & 0,00 a $A$ & 0,25 a $A$ & 0,00 a $A$ & 0,25 a $A$ & 0,00 a $A$ & 0,25 a $B$ & 0,00 a B & 0,50 a $\mathrm{BC}$ & $0,34^{\mathrm{NS}}$ \\
\hline IAC 213 & 0,25 a $A$ & 0,25 a $A$ & 0,00 a $A$ & 0,25 a $A$ & 0,00 a $A$ & 0,00 a B & 0,00 a B & 0,75 а $\mathrm{ABC}$ & $0,65^{\mathrm{NS}}$ \\
\hline IAC Caiapó & $0,00 \mathrm{~b} \mathrm{~A}$ & $0,00 \mathrm{~b} \mathrm{~A}$ & $0,00 \mathrm{~b} \mathrm{~A}$ & $0,00 \mathrm{~b} \mathrm{~A}$ & 0,25 b A & 2,00 a $A$ & 0,25 b B & $0,00 \mathrm{~b} \mathrm{C}$ & $4,63^{* *}$ \\
\hline IAC Runner 886 & 0,00 a $A$ & 0,50 a $A$ & 0,00 a $A$ & 0,00 a $A$ & 0,00 a $A$ & 0,00 a B & 1,00 a $A B$ & 0,50 a $\mathrm{BC}$ & $1,39^{\mathrm{NS}}$ \\
\hline \multicolumn{10}{|l|}{ Porte Ereto } \\
\hline IAC Tatu ST & 0,25 a $A$ & 0,00 a $A$ & 0,25 a $A$ & 0,25 a $A$ & 0,00 a $A$ & 1,25 a $A B$ & 0,75 a $A B$ & 0,00 a C & $1,89^{\mathrm{NS}}$ \\
\hline IAC 5 & 0,25 a $A$ & 0,25 a $A$ & 1,00 a $A$ & 0,00 a $A$ & 0,00 a $A$ & 0,75 a $A B$ & 0,00 a B & 0,00 a C & $1,46^{\mathrm{NS}}$ \\
\hline IAC 8112 & $0,00 \mathrm{~b} \mathrm{~A}$ & $0,25 \mathrm{~b} \mathrm{~A}$ & $0,00 \mathrm{~b} \mathrm{~A}$ & $0,00 \mathrm{~b} \mathrm{~A}$ & $0,50 \mathrm{~b} \mathrm{~A}$ & $0,75 a b A B$ & 2,00 a $A$ & $0,50 \mathrm{~b} \mathrm{BC}$ & $4,33^{* *}$ \\
\hline IAC 22 & $0,00 \mathrm{~b} \mathrm{~A}$ & 0,50 ab A & $0,75 a b A$ & 0,25 b A & 0,25 b A & 0,25 b B & $1,25 \mathrm{ab} A B$ & 1,75 a $A B$ & $3,46^{* *}$ \\
\hline IAC 88-1 & 0,25 b A & $0,00 \mathrm{~b} \mathrm{~A}$ & 0,00 b A & 0,25 b A & $0,00 \mathrm{~b} \mathrm{~A}$ & 0,25 b B & 0,25 b B & 2,00 a $A$ & $4,33^{* *}$ \\
\hline$F(D)$ & $0,16^{\mathrm{NS}}$ & $0,36^{\mathrm{NS}}$ & $1,37^{\mathrm{NS}}$ & $0,16^{\mathrm{NS}}$ & $0,31^{\mathrm{NS}}$ & $4,09 * *$ & $4,53 * *$ & $5,09 * *$ & - \\
\hline
\end{tabular}

Médias seguidas de mesma letras, minúscula na linha e maiúscula na coluna não diferem pelo teste de Tukey a $5 \%$ de probabilidade (dados foram transformados em $(x+0,5)^{1 / 2}$, para análise); **significativo a $1 \%$ de probabilidade; ${ }^{\text {Ns}: ~ n a ̃ o ~ s i g n i f i c a t i v o . ~}$ 
Tabela 4. Valores da análise de desdobramento da interação entre cultivares de amendoim versus épocas de avaliação, referentes ao número médio de brotos por planta, sob infestação natural de Stegasta bosquella em campo. Urutaí (G0), 2009/2010.

\begin{tabular}{|c|c|c|c|c|}
\hline \multirow{2}{*}{ Cultivar (C) } & \multicolumn{3}{|c|}{ Épocas de avaliação (D) } & \multirow{2}{*}{$F(C)$} \\
\hline & 30 & 60 & 90 & \\
\hline \multicolumn{5}{|l|}{ Porte Rasteiro } \\
\hline IAC 147 & 3,03 a $A$ & 3,29 a $A$ & 0,80 b BC & $247,08^{* *}$ \\
\hline IAC 213 & 2,39 a $B$ & 2,19 a DE & 0,97 b $A B$ & 78,34 ** \\
\hline IAC Caiapó & 2,68 a $A B$ & 2,81 a BC & 0,91 b $A B$ & $148,44^{* *}$ \\
\hline IAC Runner 886 & 2,92 a $A$ & 3,19 a $A B$ & 1,25 b A & $146,50 * *$ \\
\hline \multicolumn{5}{|l|}{ Porte Ereto } \\
\hline IAC Tatu ST & $1,23 \mathrm{~b} \mathrm{C}$ & 2,81 a BC & 0,97 b $A B$ & $131,56^{* *}$ \\
\hline IAC 5 & $1,10 \mathrm{~b} \mathrm{C}$ & $2,60 c C D$ & 0,77 c BC & $126,32 * *$ \\
\hline IAC 8112 & 1,05 b C & 2,22 a DE & 0,78 b BC & $77,54^{* *}$ \\
\hline IAC 22 & $1,20 \mathrm{~b} \mathrm{C}$ & 2,07 a $E$ & 0,48 c C & $83,56^{* *}$ \\
\hline IAC 88-1 & 1,05 b C & 2,94 a $A B C$ & 0,66 c BC & $197,30 * *$ \\
\hline$F(D)$ & $86,22 * *$ & $22,05^{* *}$ & $5,28 * *$ & - \\
\hline
\end{tabular}

Médias seguidas de mesma letras, minúscula na linha e maiúscula na coluna não diferem pelo teste de Tukey a $5 \%$ de probabilidade; **significativo a $1 \%$ de probabilidade; ${ }^{\text {Ns: }}$ não significativo.

Tabela 5. Valores da análise de desdobramento da interação entre cultivares de amendoim versus épocas de avaliação, referentes ao número médio de brotos por planta, sob infestação natural de Stegasta bosquella em campo. Urutaí (G0), 2010/2011.

\begin{tabular}{|c|c|c|c|c|}
\hline \multirow{2}{*}{ Cultivar (C) } & \multicolumn{3}{|c|}{ Épocas de avaliação (D) } & \multirow{2}{*}{$F(C)$} \\
\hline & 30 & 60 & 90 & \\
\hline \multicolumn{5}{|l|}{ Porte Rasteiro } \\
\hline IAC 147 & $1,50 \mathrm{~b} \mathrm{AB}$ & 1,81 a C & 0,37 c B & $72,86^{* *}$ \\
\hline IAC 213 & 1,84 b AB & 1,42 a $B C$ & $0,50 \mathrm{c} \mathrm{AB}$ & $59,49 * *$ \\
\hline IAC Caiapó & $1,80 \mathrm{~b} \mathrm{AB}$ & 2,29 a $A B$ & $0,45 \mathrm{c} \mathrm{AB}$ & $115,66^{* *}$ \\
\hline IAC Runner 886 & 1,95 a $A B$ & 2,20 a $A B C$ & 0,84 b A & $65,53 * *$ \\
\hline \multicolumn{5}{|l|}{ Porte Ereto } \\
\hline IAC Tatu ST & 1,46 b B & 1,96 a $A B C$ & $0,45 c A B$ & $75,08^{* *}$ \\
\hline IAC 5 & 2,15 a $A$ & 2,36 a $A$ & 0,32 b B & 159,31 ** \\
\hline IAC 8112 & 1,92 a $A B$ & 1,86 a BC & 0,39 b $A B$ & $99,84 * *$ \\
\hline IAC 22 & 1,81 a $A B$ & 1,95 a $A B C$ & $0,31 \mathrm{~b} \mathrm{~B}$ & $105,39 * *$ \\
\hline IAC 88-1 & 1,65 b AB & 1,96 a $A B C$ & 0,20 c B & $112,13 * *$ \\
\hline$F(D)$ & $5,92 * *$ & $3,98^{* *}$ & $3,18^{* *}$ & - \\
\hline
\end{tabular}

Médias seguidas de mesma letras, minúscula na linha e maiúscula na coluna não diferem pelo teste de Tukey a de $5 \%$ de probabilidade; ** significativo a $1 \%$ de probabilidade; ${ }^{\text {Ns}: ~ n a ̃ o ~ s i g n i f i c a t i v o . ~}$

Aos 30 DAE, IAC 5 apresentou maior emissão de broto, e IAC Tatu ST, o menor valor, sem diferir dos demais. Aos 60 DAE, IAC 5 emitiu o maior número de brotos, e IAC 147 , o menor valor. Já aos 90 DAE, IAC Runner 886 apresentou a maior emissão de brotos, diferindo de IAC 88-1, IAC 22 , IAC 5 e IAC 147, que apresentaram as menores emissôes de brotos.

Diferença entre os cultivares quanto ao número de brotos produzidos pode ser atribuída as suas diferentes arquiteturas vegetativas (LourenÇão et al., 2007) e trata-se de um importante parâmetro para discriminar genótipos resistentes (Godoy et al., 2001).

Lourenção et al. (2007), estudando a infestação de $E$. flavens em duas regióes, também constataram maiores emissóes de brotos aos 57 DAE e os maiores valores foram observados nos cultivares IAC Caiapó, Tégua e IAC Runner 886.
Já Chagas Filho et al. (2008), avaliando a resistência de cultivares de hábito de crescimento ereto, observaram maiores valores aos $22 \mathrm{DAE}$, sendo que os cultivares não apresentaram mecanismos de defesa em relação à infestação da praga, haja vista que o número de emissão de brotos apicais não aumentou com a infestação da praga.

\section{CONCLUSÃO}

Independentemente do cultivar, as maiores infestaçóes de S. bosquella ocorrem dos 53 aos 67 DAE.

O cultivar IAC Runner 886 apresenta a maior emissão de brotos apicais, uma característica desejável na defesa da planta em relação à $S$. bosquella. 


\section{REFERÊNCIAS}

BOIÇA JUNIOR, A.L.; CHAGAS FILHO, N.R.; GODOY, I.J.; LOURENÇÃO, A.L.; SOUZA, J.R. Avaliação de resistência de cultivares de amendoim de hábito de crescimento rasteiro a Enneothrips flavens Moulton (Thysanoptera: Thripidae). Arquivos do Instituto Biológico, v.79, n. 1, p.33-38, 2012.

BOIÇA JUNIOR, A.L.; RIBEIRO, Z.A.; CAMPUS, A.P.; CHAGAS FILHO, N.R. Técnica de criação e parâmetros biológicos de Stegasta bosquella em amendoim, Revista Caatinga, v.24, n.2, p.192-196, 2011.

BONDAR, G. Uma praga do amendoim Parastega (Gelechia) bosquella Chambers. Chácaras e quintais, v.38, n.5, p.5, 1928.

BRANDÃO FILHO, J.S. Doenças e pragas do amendoim. Agricultura e pecuária, v.241, p.4-5, 1943.

CAMPBELL, W.V.; WYNNE, J.C. Resistance of groundnuts to insects and mites. In: International Workshop on Groundnuts. ICRISAT, 1980, Patancheru, India. Proceedings... Patancheru, p.149-157, 1980.

CHAGAS FILHO, N.R.; BOIÇA JUNIOR, A.L.; GODOY, J.L.; LOURENÇÃO, A.L.; RIBEIRO, Z.A. Resistência de cultivares de amendoim de hábito de crescimento ereto a Enneothrips flavens Moulton (Thysanoptera: Thripidae). Arquivos do Instituto Biológico, v.75, n.2, p.149-156, 2008.

CRUZ, B.P.B.; FIGUEIREDO, M.B.; ALMEIDA, E. Principais doenças e pragas do amendoim no Estado de São Paulo. Biológico, v.28, n.7, p.189-195, 1962.

DI BELLO, M.M.; SOUZA, B.H.S.; JANINI, J.C. BOIÇA JÚNIOR, A.L. não preferência para a alimentação e antibiose em cultivares de amendoim a Stegasta bosquella (Chambers) (Lepidoptera: Gelechiidae). Semina, v.36, n.2, p.619-630, 2015.
EMBRAPA. Centro Nacional de Pesquisa de Solos. Sistema brasileiro de classificação de solos. Brasília: EMBRAPA; 1999. $412 \mathrm{p}$.

GABRIEL, D.; NOVO, J.P.S.; GODOY, I.J.; BARBOZA, J.P. Flutuação populacional de Enneothrips flavens Moulton em cultivares de amendoim. Bragantia, v.55, n.2, p.253-257, 1996.

GALLO, D.; NAKANO, O.; SILVEIRA NETO, S.; CARVALHO, R.P.L.; BATISTA, G.C.; BERTI FILHO, E.; PARRA, J.R.P.; ZUCCHI, R.A.; ALVES, S.B.; VENDRAMIN, J.D.; MARCHINI, L.C.; LOPES, J.R.S.; OMOTO, C. Entomologia agrícola. Piracicaba: FEALQ; 2002. 920p.

GODOY, I.J.; MORAES, S.A.; MORAES, A.R.A.; KASAI, F.K.; MARTINS, A.L.M.; PEREIRA, J.C.V.N.A. Potencial produtivo de linhagens de amendoim do grupo ereto precoce (Subespécie Fastigiata) com e sem controle de doenças foliares. Bragantia, v.60, n.2, p.101-110, 2001.

JANINI, J.C. Resistência de espécies silvestres de amendoim (Arachis spp.) ao ataque de Enneothripsflavens Moulton, 1941 (Thysanoptera: Thripidae) eStegasta bosquella (Chambers, 1875) (Lepidoptera: Gelechiidae). 2009. 89 f. Dissertação de mestrado (Entomologia Agrícola) - Faculdade de Ciências Agrárias e Veterinárias, Jaboticabal, 2009.

LARA, F.M. Princípios de resistência de plantas a insetos. São Paulo: Ícone; $1991.336 \mathrm{p}$.

LOURENÇÃO, A.L.; MORAES, A.R.A.; GODOY, I.J.; AMBROSANO, G.M.B. Efeito da infestação de Enneothrips flavens Moulton sobre o desenvolvimento de cultivares de amendoim, Bragantia, v.66, n.4, p.623-636, 2007.

SANTOS, R.C. (Ed.). O Agronegócio do amendoim no Brasil. Campina Grande: Embrapa Algodão; 2005. $451 \mathrm{p}$.

SICHMANN, W. Principais pragas da cultura do amendoim. Boletim do Campo, v.19, n.173, p.18-25, 1963. 